\title{
e-Beam Driven Accelerators: Working Group Summary
}

\author{
P. Muggli ${ }^{*}$, Group Leader \\ University of Southern California, Los Angeles, CA 90089 \\ J.S.T. Ng, Co-group Leader \\ Stanford Linear Accelerator Center, Menlo Park CA 94275
}

\begin{abstract}
The working group has identified the parameters of an afterburner based on the design of a future linear collider. The new design brings the center of mass energy of the collider from 1 to $2 \mathrm{TeV}$. The afterburner is located in the final focus section of the collider, operates at a gradient of $\approx \mathrm{GeV} / \mathrm{m}$, and is only about $125 \mathrm{~m}$ long. Very important issues remain to be addressed, and include the physics and design of the positron side of the afterburner, as well as of the final focus system. Present plasma wakefield accelerator experiments have reached a level of maturity and of relevance to the afterburner, that make it timely to involve the high energy physics and accelerator community in the afterburner design process. The main result of this working group is the first integration of the designs of a future linear collider and an afterburner.
\end{abstract}

\section{WORKING GROUP GOALS}

The working group charge was given as follows:

The working group (WG) will identify the critical experiments that should be performed to study the feasibility of a plasma-based afterburner for a linear collider. These include, but are not limited to, two-bunch plasma wakefield accelerator (PWFA) experiments, propagation in long plasmas leading to energy gains of the order of the incoming beam energy, stability of the propagation in long plasmas, erosion of the beam head when field ionizing over distances long compared to the incoming beam beta function, optimization of the transformer ratio and of the loading of the wake by the beam. The WG will also attempt to identify diagnostics appropriate for these experiments. Possible facilities where these experiment could be performed, and the generation of bunches suitable for PWFA experiments will be discussed. The $W G$ will also discuss the necessary advances in computational toll required to design and optimize a full-scale afterburner. The WG will attempt to define the parameters of an afterburner for an existing (SLAC) or future (NLC) collider, and possibly for a future, higher energy collider. The parameters include the incoming beam, the plasma, and the expected beam parameters at the collision point. Issues specifically related to

*muggli@usc.edu 
collisions in a particle detector, such as driver/witness beam separation, luminosity, background production will also be addressed.

The WG sessions were arranged around invited talks presenting the latest status of the research in PWFA and afterburner experiments and simulations. The invited speakers topics where then used as seeds for topical discussions about the work presented and how it relates to the afterburner concept and design. The present text summarizes the findings of the WG and whenever possible refers to the presentations given to the WG. We assume that the invited speakers also report about their work in these Proceedings, and we make reference to these reports.

The goals of the WG as defined at the beginning of the workshop were defined as:

1) To consider existing designs for a next linear collider [1] and for an afterburner [2], and to examine the parameters of both designs that need to be adjusted in order to boost the center of mass energy of the collider from $1 \mathrm{TeV}$ to $2 \mathrm{TeV}$.

2) To identify the most important issues that remain unaddressed or difficult in the design of the afterburner.

3) To propose the next relevant experiments which need to be performed to push forward the development of an afterburner.

In the remainder of the text we will refer to the next linear collider as nLC to distinguish the design considered here from the $11.4 \mathrm{GHz}$ based design of a $\mathrm{nLC}$ knows as NLC.

\section{NEW AFTERBURNER DESIGN}

\section{nLC Designs}

Two designs for the nLC are considered here and the full list of their parameters can be found in the Second Report of the International Linear Collider Technical Review Committee [1]. A selected list of parameters for these two designs is given in Table 1. The first design known as the NLC design is characterized by room temperature accelerating cavities excited by $\mathrm{RF}$ at $11.4 \mathrm{GHz}$ (X-band) and is also known as the "warm" design. The second design known as the US SC design is characterized by superconducting accelerating cavities excited by RF at $1.3 \mathrm{GHz}$ (Lband) and is also known as the "cold" design. The main differences between the two designs when considering their use to seed an afterburner accelerator are the slightly larger charge per bunch, and longer spacing between micro bunches in the US SC design, both a consequences of its lower RF frequency. Also, The US SC bunches are slightly longer, leading to a slightly longer bunches and delay between the driver and production bunch in the afterburner, and therefore to slightly lower plasma density and gradient. However, the two corresponding afterburner designs are very similar, and the remainder of this summary will be focused on the warm design afterburner. From a collider point of view, the US SC design reaches a slightly higher luminosity than the NLC design. In both cases the effective accelerating gradient is $<60 \mathrm{MV} / \mathrm{m}$, and leads to single linac lengths, for electrons $\mathrm{e}^{-}$or positrons $\mathrm{e}^{+}$, of $\approx 14 \mathrm{~km}$. Each linac is followed by a final focus section about $4 \mathrm{~km}$ long. Both $\mathrm{nLC}$ are preceded by L-band 
accelerator sections where the bunch train is generated and accelerated to $6 \mathrm{GeV}$. Electron and positron bunch trains are sent into damping rings at the $2 \mathrm{GeV}$ point to reach the emittances required to achieve the desired luminosity.

\section{Previous Afterburner Designs}

The design of an afterburner based on the parameters of the Stanford Linear Collider (SLC) is presented in [2]. The parameters of that design allow for the doubling of the energy of the $50 \mathrm{GeV}$ beams in two sections of plasma. The charge available from the SLC linac ( $4 \square 10^{10}$ particles/bunch) is divided between a high charge driver bunch and a low charge witness (or production) bunch in a 3:1 ratio. The bunches are compressed to Gaussian rms longitudinal sizes $\square_{\mathrm{z}}$ of 62 and $30 \mu \mathrm{m}$ for the driver and production bunches respectively, and are separated by $\approx 180 \mu \mathrm{m}$. The corresponding plasma density is $1.8 \square 10^{16} \mathrm{~cm}^{-3}$, resulting in an accelerating gradient of $7 \mathrm{GeV} / \mathrm{m}$ in the electron beam case. The energy of the incoming electron bunch can therefore be doubled in $\approx 7 \mathrm{~m}$ of plasma. The accelerating PWFA sections are immediately followed by thin $(2 \mathrm{~mm})$ plasma lenses with a density in the $2 \square 10^{18} \mathrm{~cm}^{-3}$ range to focus the beams at the interaction point to $\approx 100 \mathrm{~nm}$ radius to compensate for the loss of luminosity resulting from the splitting of the total charge available. The positron side of the afterburner has parameters similar to those of the electron side. However, numerical simulations indicate that the accelerating gradient excited by a $\mathrm{e}^{+}$ bunch is smaller than that excited by an $\mathrm{e}^{-}$bunch with similar parameters, and that the gradient can be enhanced by using a hollow plasma channel. The $\mathrm{e}^{+}$section of the afterburner is therefore slightly longer ( $\approx 10 \mathrm{~m}$ ) and includes a hollow plasma channel.

\section{New Afterburner Design}

The design presented here after is based the design presented by $\mathrm{T}$. Raubenheimer in his tutorial talk and invited talk to the working group [3].

The bunch trains suitable for the afterburner are obtained by generating two bunch trains with a pattern identical to these in the nLC designs, i.e., spaced by 2.8 or $337 \mathrm{~ns}$. Note that the longer spacing between bunches would allow for better feedback systems and would also provide more time for the plasma to recover from the previous bunches wake and energy deposition. These two bunch trains are each accelerated to an energy of $6 \mathrm{GeV}$, with a relative charge of $\approx 3: 1$, or 1.1 and $0.4 \square 10^{10}$ particles per respective bunch (see Fig.1) in the S-band linac. The total charge is limited by beam loading in the RF accelerator. At the $2 \mathrm{GeV}$ point, the production bunch train is sent into a damping ring to reach the small emittances required for the high luminosity. After the damping ring the production bunches are compressed, and compressed again at the end of the S-band linac to $\square_{z}=30 \mu \mathrm{m}$. The high charge driver bunches need not to have ultra-low emittances and are therefore directly compressed to $\square_{\mathrm{z}}=62 \mu \mathrm{m}$. Positron bunches with similar characteristics can be generated using the method described in the nLC design. The two bunch trains are then interlaced with a delay between respective bunches of 0.6 ps or $180 \mu \mathrm{m}$, appropriate for a plasma density of $\mathrm{n}_{\mathrm{e}}=1.8 \square 10^{16} \mathrm{~cm}^{-3}$. The interlaced bunch trains are accelerated to $500 \mathrm{GeV}$ in the RF linac described in [1]. 
The afterburner plasma sections are located in the respective final focus regions of the nLC. Before the plasma sections both bunch trains transverse sizes are then adjusted have equal beta functions in both planes $\left(\square_{x}=\square_{y}=5.3 \mathrm{~cm}\right)$. This is required for the matching of the beams to the afterburner plasma. The matched transverse sizes are given by:

$$
\square_{x, y, \text { match }}=\frac{\square\left(W_{x, y}\right.}{\square n_{e} \square} \frac{2 \square}{r_{e}}
$$

where $\mathrm{r}_{\mathrm{e}}=\mathrm{e}^{2} / 4 \square \mathrm{mc}^{2} \approx 2.8 \square 10^{-15} \mathrm{~m}^{-3}$ is the classical electron radius. For the beam parameters of Table 1, the matched sizes for the production bunches are $\square_{\mathrm{x}, \text { match }}=0.440 \mu \mathrm{m}$ and $\square_{\mathrm{y}, \text { match }}=0.045 \mu \mathrm{m}$. The emittances of the driver bunches may be two to four orders of magnitude larger if these were not send through damping rings. Their matched sizes may therefore be one to two orders of magnitude larger than that of the production bunch. The plasma can tolerate larger sizes as long as they remain small compared to the plasma collisionless skin depth $\mathrm{c} / \square_{\mathrm{p}} \approx 40 \mu \mathrm{m}$ at $1.8 \square 10^{16} \mathrm{~cm}^{-3}$. Additional emittance damping may be required if that condition was not satisfied, and a pre-damping ring may be necessary for the positron beam.

In the afterburner the production bunch is accelerated while the driver bunch is decelerated. As the production bunch energy increases, its matching condition could

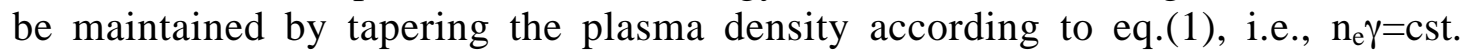
Betatron oscillations of the driver bunches envelope is not of concern since they are not participating in the final collision process. On the other hand, driver bunch head erosion could delay the wake formation and could be compensated for by increasing the plasma density along the plasma length. The best solution for the plasma density profile along the afterburner plasma section, also including stability against the hose instability [4], will have to be provided by full-scale simulations of the acceleration process.

Scaling of the bunch transverse field to these used in the E-164/E-164X experiments $[5,6]$ indicates that for the afterburner described here the plasma can be created by field ionization of an $\mathrm{H}_{2}$ gas. In such a system the plasma density is equal to the neutral gas density, is constant in time and can be set very accurately. The low atomic number of hydrogen also minimizes the collision probability between beam and plasma/neutral particles, and completely eliminates the possibility of additional impact- or field-ionization because of the wake fields or beam dynamics. The plasma length is limited only by erosion of the driver bunch head.

The plasma density is estimated from the bunch spacing $(180 \mu \mathrm{m})$ and is of the order of $1.8 \square 10^{16} \mathrm{~cm}^{-3}$. At this density the acceleration gradient is $\approx 4 \mathrm{GeV} / \mathrm{m}$, and the plasma length is therefore $125 \mathrm{~m}$ in order for the production bunch to gain $500 \mathrm{GeV}$.

At the plasma exit, the driver bunches energy spectrum spreads between the incoming energy of $500 \mathrm{GeV}$ and $\approx \mathrm{GeV}$. This is due to the fact that the particles located very early in the driver bunch travel in a neutral plasma and are essentially not affected by it, while the core particles experience the full decelerating field. At this point, systems designed to dump other particles and radiation generated in the plasma are used to clean the beam for the interaction in the particle detector. The production bunch parameters such as position, pointing, energy and energy spread can also be measured and used for feedback and feedforward action on the following bunches in a 
single train, as well as on following trains. Finally the bunch can be conditioned for the final focus plasma lens.
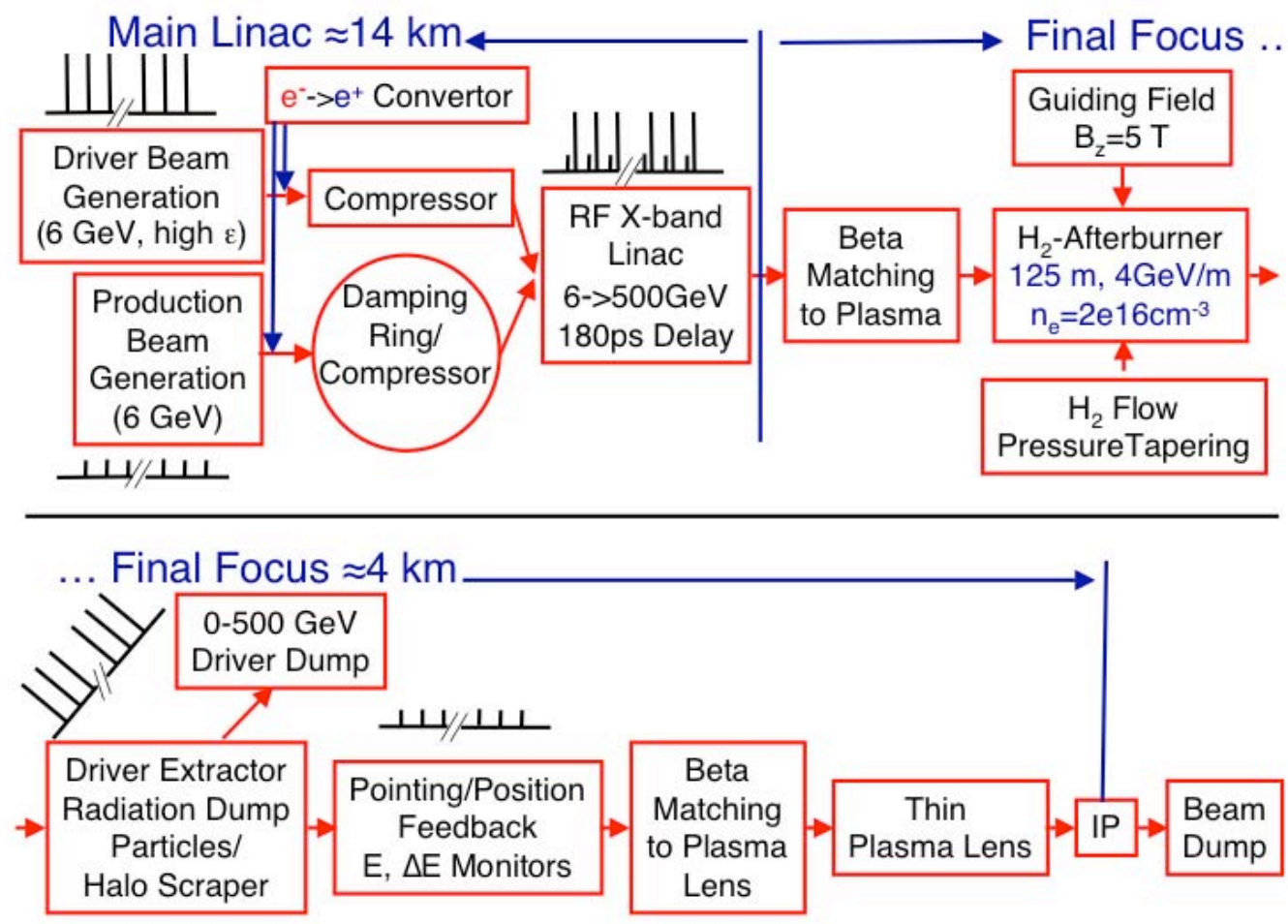

FIGURE 1. Flowchart of the RF linac and afterburner. The bunch patterns are also sketched (not to scale). Note that in the actual design the damping ring and compressor are located at the $2 \mathrm{GeV}$ point of the $6 \mathrm{GeV} \mathrm{S}$-band linac. In the present flow chart they are put after the $6 \mathrm{GeV}$ linac for simplicity.

The design of the plasma lens remains a challenge. So far no experiment has demonstrated focusing to the sub-micron sizes called for in order to reach the desired luminosity. Assuming that the plasma section preserves the emittance of the incoming production bunch, calculating the plasma density required to focus the beam from its size at the plasma exit to the size at the IP from $K=1 / \square_{\text {matched }} \square_{\mathrm{IP}}$ (where $\mathrm{K}=\square_{\mathrm{pe}}{ }^{2} / 2 \mathrm{c}^{2}$ is the plasma restoring term in the beam envelope equation, $\square_{\mathrm{p}}=\left(\mathrm{n}_{\mathrm{e}} \mathrm{e}^{2} / \square \mathrm{m}\right)^{1 / 2}$ is the electron plasma angular frequency), and leads to $n_{e} \approx 1.6 \square 10^{12} \mathrm{~cm}^{-3}$. However at this plasma density the mean ion interspacing is $\approx_{\mathrm{e}}{ }^{1 / 3} \approx 0.85 \mu \mathrm{m}$, i.e., larger than the beam size. This plasma lens is therefore not practical for the final focus.

The WG assumed that the transformer ratio of the plasma accelerating section is $\approx 1$, and the efficiency of energy transfer from the driver to the production bunch is therefore simply given by the ratio of the number of particles in each bunch: $0.4 / 1.1 \approx 36 \%$. The wall plug to beam efficiency is therefore reduced by a minimum of $74 \%$. A coarse estimate of the energy remaining in each driver bunch is obtained by assuming that about half the energy no transferred to the production bunch or about $310 \mathrm{~J}$ remain in the bunch, corresponding to an average power of $\approx 3.6 \mathrm{MW}$, 
significantly less than the original design for the full beam dump power of $\approx 15 \mathrm{MW}$. This coarse energy balance also shows that a significant amount of energy is deposited in the plasma, $\approx 3.6 \mathrm{MW}$ or $\approx 29 \mathrm{~kW} / \mathrm{m}$. Although this power level does not represent a cooling issue, the resulting local plasma temperature is of concern. The energy is deposited in a radius of plasma approximately equal to a collisionless skin depth $\mathrm{c} / \square_{\mathrm{p}} \approx 40 \mu \mathrm{m}$ at $\mathrm{n}_{\mathrm{e}}=1.8 \square 10^{16} \mathrm{~cm}^{-3}$. A single driver bunch therefore transfers about $130 \mathrm{keV}$ to each plasma electron. This will lead to significant local plasma heating, ion motion, and reduction of the density on the beam axis after the bunch(es) passage. This deleterious effect can be alleviated by flowing the $\mathrm{H}_{2}$ gas. The required flow velocity is of the order of a distance of two plasma skin depths in the time between two bunches or $64 \mu \mathrm{m} / 2.8 \mathrm{~ns} \approx 2.45 \square 10^{4} \mathrm{~m} / \mathrm{s}$. The flow velocity would be much lower in the US SC bunch spacing case (337 ns). Flowing the gas and therefore the plasma would also remove the remaining wakefields for the subsequent bunch.

TABLE 1. NLC and TESLA Parameters (reproduced from T. Raubenheimer, these

\begin{tabular}{|c|c|c|c|c|}
\hline \multicolumn{5}{|l|}{ Proceedings) } \\
\hline \multirow[t]{2}{*}{ Final Energy } & \multicolumn{2}{|c|}{$2 \mathrm{TeV}$} & \multicolumn{2}{|c|}{$2 \mathrm{TeV}$} \\
\hline & NLC & Afterburner & US SC & Afterburner \\
\hline CM Energy $(\mathrm{GeV})$ & 1000 & 2000 & 1000 & 2000 \\
\hline Linac Length $(\mathrm{km})$ & 14.1 & 0.13 & 30 & 0.21 \\
\hline Repetition Rate (Hz) & 120 & 120 & 5 & 5 \\
\hline Bunch Charge $\left(10^{10}\right)$ & 1.5 & $1.1 / 0.4$ & 2 & $1.5 / 0.5$ \\
\hline Bunches per RF Pulses & 96 & & 2820 & \\
\hline Bunch Separation & $2.8 \mathrm{~ns}$ & $0.6 \mathrm{ps}$ & $337 \mathrm{~ns}$ & $1 \mathrm{ps}$ \\
\hline Effective Gradient (MV/m) & 52 & 4000 & 35 & 2400 \\
\hline Plasma Density $\left(\mathrm{cm}^{-3}\right)$ & & $2 \square 10^{16}$ & & $9 \square 10^{15}$ \\
\hline Ш at IP (m-rad) & $360 \square 10^{-8}$ & $360 \square 10^{-8}$ & $960 \square 10^{-8}$ & $960 \square 10^{-8}$ \\
\hline Ш, at IP (m-rad) & $4 \square 10^{-8}$ & $4 \square 10^{-8}$ & $4 \square 10^{-8}$ & $4 \square 10^{-8}$ \\
\hline $\begin{array}{l}\text { Beam Area Reduction by the } \\
\text { Plasma Lens }\end{array}$ & & 10 & & 11 \\
\hline$\square_{\mathrm{x}} / \square_{\mathrm{y}}$ at IP $(\mathrm{nm})$ & $219 / 2.1$ & $37 / 3.9$ & $489 / 4.0$ & $67 / 4.3$ \\
\hline$\square_{\mathrm{z}}$ at IP $(\mu \mathrm{m})$ & 110 & 32 & 300 & 35 \\
\hline$\square_{\text {ave }}$ & 0.27 & 5 & 0.11 & \\
\hline Pinch Enhancement & 1.4 & 1.1 & 1.7 & 1 \\
\hline Beamstrahlung $\square \mathrm{B}(\%)$ & 8.4 & 40 & 5.9 & 32 \\
\hline Photons per $\mathrm{e}^{-} / \mathrm{e}^{+}$ & 1.2 & 2 & 1.6 & 10 \\
\hline Luminosity $\left(10^{33}\right)$ & 31 & 10 & 38 & 10 \\
\hline
\end{tabular}

The issue of scattering of the production bunch particles on the $\mathrm{H}$ nuclei is of concern since it can cause emittance growth, particles scattering and loss, beam halo formation, and radiation production. However, the conclusion reached based on an evaluation for a $10 \mathrm{GeV}$ to $1 \mathrm{TeV}, \mathrm{n}_{\mathrm{e}}=10^{17} \mathrm{~cm}^{-3}, 6 \mathrm{GeV} / \mathrm{m}$, plasma beat wave accelerator published in ref. [7] is that the calculated emittance growth is $\approx 10^{-8} \mathrm{~m}$-rad. With this relatively small emittance growth, Table 1 indicates that with the parameters of the design presented here the energy of the collider can be doubled with a loss of luminosity by a factor of only about three. However, the energy doubling is achieved without doubling the length of the collider.

Relatively little is known about positron PWFA's. It has been demonstrated experimentally that $\mathrm{e}^{+}$can be focused $[8,9]$ and accelerated [ 10$]$ by plasmas. 
Experimental and numerical results indicate that because of nonlinear plasma focusing force emittance growth is expected in a $\mathrm{e}^{+}$PWFA [11]. Simulation results also indicate the accelerating gradient driven by a $\mathrm{e}^{+}$bunch is smaller than that driven by an $\mathrm{e}^{-}$ bunch with similar parameters [12], but that the gradient can be increased by $\approx 100 \%$ by using a hollow plasma channel. Recent results also indicate that when the $\mathrm{e}^{+}$bunch creates its own plasma by field-ionization, its accelerating wake is also slightly larger than that in a pre-ionized plasma. However, the WG assumed that the positron side of the afterburner would lead to similar performances as the electron side. At this time, it is clear that more experimental, simulation and theoretical results about the $\mathrm{e}^{+}$PWFA are needed and that the realization of the $\mathrm{e}^{+}$half of the afterburner maybe more complicated than its $\mathrm{e}^{-}$half.

\section{AFTERBURNER DESIGN ALTERNATIVES}

The WG considered a number of alternatives to the above design. These alternatives would lead to more complex practical realizations of the afterburner, but may be required to reach the required beam parameters at the interaction point and could lead to a more efficient afterburner.

Most of the issues related to the $\mathrm{e}^{+}$half of the afterburner could be avoided by using an $\mathrm{e}^{-}$bunch driver for the $\mathrm{e}^{+}$production bunch. In this case the driver e- bunch driver and the $\mathrm{e}^{+}$production bunch would have to be accelerated in separate 6 to $500 \mathrm{GeV}$ lines. In the plasma accelerator section the $\mathrm{e}^{+}$bunch would be placed at the plasma wake phase where the fields are both focusing and accelerating. Simulation studies are necessary to validate this possibility and to determine the quality of the $\mathrm{e}^{+}$bunch at the plasma exit.

The wall plug efficiency could be improved by splitting the $125 \mathrm{~m}$ plasma accelerating section in $13,10 \mathrm{~m}$ sections each driven by a $50 \mathrm{GeV}$ beam. This alternative would also allow for monitoring and feedback devices to be inserted between each plasma sections, potentially leading to more stable beam parameters at the IP. However, this alternative also creates a significantly longer and more complex and after-burner sections.

\section{PROGRESS TOWARD AN AFTERBURNER}

The presentations to the WG showed that three majors steps toward the realization of an afterburner have been realized since AAC'02. The first step is the replacement of a pre-ionized plasma by a field-ionized plasma created by the driver bunch itself. This new regime of the PWFA was reached for the first time in the SLAC E-164/E-164X experiments [5]. It allows in principle to produce high-density plasmas $\left(>10^{16} \mathrm{~cm}^{-3}\right)$ over long distances, plasmas of the type needed for the afterburner. The energy lost to ionization is small compared to the bunch particles energy ( $\approx 13 \mathrm{MeV} /$ particles for the driver bunch to form a plasma with a radius of $\mathrm{c} / \square_{\mathrm{p}}$ at $\mathrm{n}_{\mathrm{e}}=2 \square 10^{16} \mathrm{~cm}^{-3}$ over $125 \mathrm{~m}$ of hydrogen with an ionization potential of $13.6 \mathrm{eV}$ ), the plasma density is fully determined by the gas pressure and can be easily tapered if needed. 
The second step is the experimental demonstration of the excitation of accelerating gradients of $\approx 40 \mathrm{GeV} / \mathrm{m}[6,13]$, larger than those envisaged for the afterburner. This remarkable result was made possible by the recent availability of short, high current electron bunches at the Stanford Linear Accelerator Center.

The third step is the writing of a reduced algorithm PIC code called QuickPIC that was used to simulate an SLC-based energy doubler in a reasonable amount of CPU time [14]. This code is the first to open the possibility for the accurate simulation and design of the afterburner sketched here, something impossible with full algorithms codes available until now.

Presentations to the working group also showed an important effort to include more physics in the numerical codes used to design and describe PWFA experiments. The effect that need to be evaluated in the design of an afterburner include field, and impact ionization, scattering on the plasma ions, as well as radiation by the bunch particles (betatron, bremstahlung, etc.) $[15,16]$.

\section{SUGGESTED EXPERIMENTS}

The WG identified a number of key experiments that should be performed in the near future to better assess the parameters and features of a an afterburner for a nLC. A list of these experiments is given in this section.

The recent high gradient, large energy gain experimental results [6, 13] were performed with a plasma length of $\mathrm{L} \approx 10 \mathrm{~cm}$ and need to be repeated over longer plasma lengths. This would demonstrate that these types of gradient can be sustained over long distances, and would allow for the study the other issues related to the propagation in long plasmas, in particular head erosion (for $\mathrm{L}>>\square_{\mathrm{x}, \mathrm{y}}$ ), and hose stability (for $L>>\square_{\square}=\left(2 \square^{1 / 2} \mathrm{c} / \square_{\mathrm{p}}, \square_{\square}\right.$ is the betatron wavelength). This experiment can only be performed at the Stanford Linear Accelerator Center (SLAC), the only facility where ultra-short, ultra-relativistic electron bunches are available. However, such an experiments needs a new beam dump with an appropriate energy acceptance.

The growth rate $\mathrm{G}$ of the hose instability [4] is proportional to the number of betatron oscillations of the beam envelope over the plasma length: $\mathrm{G} \sim\left(\mathrm{L}^{2} \square_{\mathrm{z}} / \square_{\square}\right)^{1 / 3} \sim\left(\mathrm{L}^{2} \square_{\mathrm{z}} / \square^{1 / 3}\right.$. Experiments devised to validate the numerical tools that are used to predict the stability of the afterburner bunches against the hose instability can therefore be scaled to lower energy, longer bunches and shorter plasma length.

Most PWFA accelerator experiments use a single electron bunch to excite the wake and experience the acceleration, and result in very broad particle energy spectra. Experiments need to be performed with two bunches, a driver and a witness bunch. These would demonstrate the acceleration of a particle beam with a finite energy spread and emittance, similar to the ones obtained in numerical simulations of the afterburner [14]. Narrow energy spreads can only be achieved through beam loading of the plasma wake. Preliminary experiments are currently performed at the A0 facility [17], and an ideal facility for such experiments could become available at the SLAC/ORION facility [18]. Desired parameters for such an experiments would be: a driver with an energy of a few hundred $\mathrm{MeV}$ and $\leq 100$ ps to allow for energy gains in the $100 \mathrm{MeV}$ range, and a lower energy witness bunch, shorter than the driver bunch, 
with a variable charge and delay with respect to the driver bunch. Experiments with a beam pre-bunched at the $10 \mu \mathrm{m}$ scale are planed at the Brookhaven ATF [19]. Experiments performed by the UCLA/NICADD group study the possibility of creating a short trailing bunch using a plasma density step [20]. However, the emittance of such a trapped trailer bunch may be too large for high-energy physics applications.

Bunch shaping can be used to increase the transformer ratio and therefore the efficiency of the PWFA section of the afterburner. It could also be used to mitigate the hose instability and minimize head erosion effects [14]. Electron bunch shaping experiments are currently performed at the Neptune-UCLA laboratory [21].

The afterburner design calls for particularly small beam sizes at the IP. Plasma lenses have the advantage of particularly large focusing strength when compared to conventional focusing elements (magnets). However, no plasma lens to date has demonstrated sub-micron focused sizes. Plasma lens experiments are therefore required to explore the suitability of plasma lenses for such applications.

Note that most of the experiments proposed here can be performed either with beams with $\mathrm{GeVs}$ of energy or at lower energy $(\mathrm{MeV})$. However, the WG recognized that many of the significant results have been achieved because facilities with appropriate beam parameters became available. This is especially true in the case of the new field-ionized PWFA regime, and the large gradient and energy gain that were made possible only because of the recently short electron bunches available at SLAC, and evident in the case of the studies necessary for the positron section of the afterburner. While in the conventional section of a linear collider positrons can be handled in ways symmetric to the ways electrons are handled, this is not the case in a plasma section. In the plasma, electrons are the mobile species, and respond to the charge sign of the bunch. This leads to asymmetries that make it necessary to find solutions specific for each charge species, and to repeat experiments with bots species.

Finally, the WG group also recognized that many of the progress and research presented in the other WG are key to the realization of an afterburner and refers the reader to the corresponding reports in these Proceedings. These include beam diagnostics, beam manipulation and synchronization, as well as simulation capabilities.

\section{CONCLUSIONS}

The WG has identified the parameters of an afterburner based on the design of a future linear collider. The design is based on the parameters of the $1 \mathrm{TeV}$ upgrade to a next linear collider as defined in the International Linear Collider Technical Review Committee Second Report (2003) [1] and is the first attempt to merge a next linear collider and a plasma afterburner design [2]. The new design brings the center of mass energy of the collider $2 \mathrm{TeV}$. The bunch trains appropriate for the PWFA section are generated by adding a second $0-6 \mathrm{GeV}$ L-band section in the front sections of the collider. The afterburner is located in the final focus section of the collider, operates at a gradient of $\approx 4 \mathrm{GeV} / \mathrm{m}$, and is only about $125 \mathrm{~m}$ long. The plasma is created by field ionization of a hydrogen gas. Experiments with electron PWFA have reached parameters that are comparable to those proposed in the design presented here. 
However, experiments demonstrating acceleration at large gradient over long plasmas, acceleration of a witness bunch, and stability against hose instability are needed. Many of these experiments can be conducted a MeV energies. After-burner parameters can only be reached with multi-GeV beams, and it is very important that such a facility be available in the future to demonstrate experimentally the viability of the afterburner concept. Very important issues remain to be addressed, and include the physics and design of the positron side of the afterburner, as well as of the final focus system. The PWFA experiments have reached a level of maturity and of relevance to the afterburner, that make it timely to involve the high-energy physics and accelerator community in the afterburner design process. The main result of this working group is the first integration of the designs of a future linear collider and an afterburner.

\section{ACKNOWLEDGMENTS}

The authors would like to acknowledge the many contributions of the working group sessions participants. It is their contributions and their enthusiasm that made the workshop interesting and productive. The present summary is intended to reflect their contributions. However, possible mistakes and contradictions appearing in the present text are the sole responsibility of the authors.

\section{APPENDIX}

WG Tutorial Talks:

C. Joshi, UCLA- Review of Experimental Results in Electron-Driven Plasma-Wake Acceleration

T. Raubenheimer, SLAC-Afterburner at SLAC - the Collider Point of View

\section{Related Tutorial/Invited Talks}

G. Dugan, Cornell Univ.- Advanced Accelerator System Requirements Overview

Warren Mori, UCLA- Advances in Simulation Capabilities

J. Rosenzweig, UCLA- Recent Developments in Electron Bunch Compression

\section{Working Group Invited Talks:}

C. Huang, UCLA-Simulation Studies of an Afterburner Conceptual Design

T. Raubenheimer, SLAC: - Afterburner-related Issues for Linear Colliders

C. O'Connell, SLAC-Field Ionization of a Neutral Lithium Vapor using a 28.5

$\mathrm{GeV}$ Electron Beam

M. Hogan, SLAC- Energy Gain in E-164X

E. Colby, SLAC-Potential Beams at ORION

$V$. Yakimenko, BNL-Acceleration and Focusing of Relativistic Electrons in Overdense Plasma

Matt Thompson, UCLA- The UCLA/NICADD Plasma Density Transition Trapping Experiment 


\section{Contributing Talks:}

$W$. Lu, UCLA- Theory for linear and nonlinear plasma wakes driven by electron beams

M. Zhou, UCLA- QuickPIC simulations of electron/positron beam propagation in meters long plasmas--hosing instability concerns

N. Barov, NIU -Beam-plasma Experiments at FNAL

C. Barnes. SLAC-Hosing instability in the E-164X experiment?

Talks in the Joint Session with the Computational Accelerator Working Group:

D. Dimitrov, TechX Corp.- The IONPACK Library of Ionization Algorithms for PIC Codes

J. Rosenzweig, UCLA- Energy Loss of a High Charge Bunched Electron Beam in Plasma: Simulations, Scaling, and Accelerating Wake-fields

D. Bruhwiler, TechX Corp.- Simulation of Ionization Effects for High-Density Positron Drivers in future Plasma Wakefield Experiments

\section{REFERENCES}

1. International Linear Collider Technical Review Committee, Second Report, 2003, available at http://www.slac.stanford.edu/xorg/ilc-trc/2002/2002/report/PAPERS/TRC03PR.PDF

2. S. Lee et al., Phys. Rev. ST Accel. Beams 5, 011001 (2002).

3. T. Raubenheimer, these Proceedings.

4. A. Geraci and D. H. Whittum, Phys. Plasmas 7, 2241 (2000)

5. C. O.Connell et al., these Proceedings.

6. M.J. Hogan et al., these Proceedings.

7. B.W. Montague and W. Schnell, Proc. of the Malibu Conf., p. 146, (1985).

8. J.S.T. Ng, et al. Phys. Rev. Lett. 87, 244801 (2001).

9. M.J. Hogan et al. Phys. Rev. Lett. 90, 205002 (2003).

10. B.E. Blue et al., Phys. Rev. Lett. 90, 214801 (2003).

11.P. Muggli et al., to be published.

12. S. Lee et al, Phys. Rev. E 64, 045501 (2001).

13. C. Joshi et al., these Proceedings.

14. C. Huang et al., these Proceedings.

15. D. Bruhwiler et al., these Proceedings, and Proc. Part. Accel. Conf. 2003, May 12-16, Portland, OR, 734, (2003).

16. D. Dimitrov et al., these Proceedings.

17. N. Barov et al., these Proceedings, and Phys. Rev. ST Accel. Beams 7, 061301 (2004).

18. E. Colby et al., these Proceedings.

19. V. Yakimenko et al., these Proceedings, and Phys. Rev. Lett. 91 , 014802 (2003).

20. M. Thompson et al., these Proceedings, and Phys. Rev. ST Accel. Beams 7, 011301 (2004)

21. J. Rosenzweig et al., these Proceedings. 